MR-10815-178

\title{
CONTRACT STATUS REPORT FOR \\ MHD RESEARCH AND DEVELOPMENT AND OPERATION OF THE MHD COAL FIRED FLOW FACILITY
}

For The Month Ending

June 30, 1995

Work Performed Under Contract No. DE-AC02-79ET10815

\author{
Prepared by \\ The University of Tennessee \\ Space Institute \\ Energy Conversion Research \& Development Programs
}

For

The United States Department of Energy DISTRIBUTION OF THIS DOCUMENT IS UNLIMITED 


\section{DISCLAIMER}

This report was prepared as an account of work sponsored by an agency of the United States Government. Neither the United States Government nor any agency thereof, nor any of their employees, make any warranty, express or implied, or assumes any legal liability or responsibility for the accuracy, completeness, or usefulness of any information, apparatus, product, or process disclosed, or represents that its use would not infringe privately owned rights. Reference herein to any specific commercial product, process, or service by trade name, trademark, manufacturer, or otherwise does not necessarily constitute or imply its endorsement, recommendation, or favoring by the United States Government or any agency thereof. The views and opinions of authors expressed herein do not necessarily state or reflect those of the United States Government or any agency thereof. 


\section{DISCLAIMER}

Portions of this document may be illegible in electronic image products. Images are produced from the best available original document. 


\section{PROJECT STATUS REPORT}

1. CONTRACT OBJECTIVE - No change since last report.

\section{TECHNICAL APPROACH CHANGES - None}

3. CONTRACT TASKS - In this report, UTSI reports on the status/progress by program tasks identified in Chapter 3 (Work Plan) of the draft Contract Management Plan for the FY95 period, which was submitted to DOE February 17, 1995.

\section{TASK I-CONTRACT MANAGEMENT AND CLOSEOUT}

The 33rd Symposium on Engineering Aspects of Magnetohydrodynamics hosted by UTSI and ERC, Inc. was held June 12-15, 1995.

In response to a DOE/PETC letter of $5 / 23 / 95$, work continued on the development of a contract proposal package for "UTSI/CFFF MHD Program Completion and Related Activity."

The contract status report for May 1995 was issued to DOE.

Personnel staffing for the month was 20.2 equivalent full-time (EFT) personnel versus a planned level of $26.3 \mathrm{EFT}$.

\section{TASK \| - FACILITY AND PROPERTY DISPOSITION}

Facility activities this month were confined to the continuation of preventative maintenance and repairs required to maintain the Coal-Fired Flow Facility (CFFF) in a standby condition.

The reconditioning of the CFFF process water cooling tower continued. An interior protective polyurethane coating was applied on the bottom of each cooling tower quadrant.

Semi-annual maintenance on the fire protection system was initiated.

Control of government property and the maintenance of records were continuing. 
TASK III - DATA REPORTING AND ARCHIVING

Preparation of topical reports pertaining to the MHD POC test program continued.

Measurements and evaluations of corrosion from superheater test module tube samples from test section 1 and 2 were completed and preparation of a topical report on the findings is underway.

All data and documentation developed thus far on the MHD/CFFF project and identified for archiving are continuing to be maintained for archiving storage.

\section{TASK IV - SITE ENVIRONMENTAL COMPLIANCE AND REMEDIATION}

Under a subcontract for the first round of groundwater samples which will be used as a screening technique to verify what parameters must be analyzed in the other six wells that will be installed. This technique will save funds by eliminating all groundwater pollutants except those detected above drinking water standards.

Groundwater compliance monitoring work has basically come to a halt due to severe funding limitations.

Monitoring of the holding pond effluent continued in order to comply with the UTSI water discharge permit. Water is checked for $\mathrm{pH}$, temperature, flow rate, oil and grease content, total dissolved solids, and total suspended solids. All parameters continue to be measured by the UTSI chemistry lab. Any changes in CFFF operation might require monitoring of additional parameters.

\section{OPEN ITEMS}

A. DOE: The following reports/documents were in DOE review for comments and/or approval as of June 30, 1995.

FY1995 Management Plan

UTSI Itr 02/17/95

B. UTSI: The following actions were in process or preparation as of June30, 1995.

Superheater/Air Heater Tube Corrosion Studies

Est. Completion 09/30/95

$\mathrm{NO}_{x}$ Formation and Control, Topical Report

Est. Completion 07/30/95

Western Coal Processing System, Topical Report

Est. Completion 07/30/95

Western Coal 1,000 Hr POC \& Final Summary Report

Est. Completion 07/30/95 


\section{SUMMARY STATUS ASSESSMENT AND FORECAST}

The CFFF continues in standby condition as preventive maintenance and repairs are accomplished as needed.

Efforts on the preparation of topical reports continued. Work has been extended on these reports due to limited manpower resources available. 


\begin{tabular}{|c|c|c|c|c|c|c|c|c|c|c|c|c|c|c|c|}
\hline & & & Mile & one & Scl & redu & le $a n$ & $d S t$ & atus & $\operatorname{Re}$ & oort & & & & \\
\hline & $\begin{array}{l}\text { intract Identification } \\
\text { OPERA }\end{array}$ & N OF TH & COAL-F & DLOWF & :LLTY & & $\begin{array}{l}\text { 2. Reportli } \\
\text { June } 1 .\end{array}$ & $\begin{array}{l}\text { Pertod } \\
1995 \text { - June }\end{array}$ & 30,1995 & & 3. Contract Nu & & $E-A C O 2 \cdot \pi$ & 79ET10815 & \\
\hline & ntractior: Nama, Address & $\begin{array}{l}\text { UNIVER: } \\
\text { TIUTE } \\
\text { TAHOMA }\end{array}$ & $\begin{array}{l}\text { TY OFT } \\
\text { TENNES }\end{array}$ & $\begin{array}{l}\text { ESSEE S } \\
37388\end{array}$ & & & 5.contrac & $\begin{array}{l}\text { Star Dato } \\
10-01 \cdot 7\end{array}$ & & & \begin{tabular}{|l}
6. Contract Co \\
\end{tabular} & $\begin{array}{r}\text { mplation D: } \\
s\end{array}$ & pptember 30 & 30,1995 & \\
\hline 7 & Aeporting Calegory - & & & & & & FY: & & & & & & & FY95 Co & osts* \\
\hline $\mid \begin{array}{c}\mathrm{s} \\
\mathrm{k}\end{array}$ & $\begin{array}{l}\text { Breagdownn Sirvature } \\
\text { Element }\end{array}$ & Oct & Nov & Dec & Jan & Fab & Mar & Apr & May & Jun & Jul & Aug & Sep & Accrued & EAC* \\
\hline 1 & & & $\overline{B V}$ & 87 & Bत्रण & $\bar{A} \nabla \mathbf{B}$ & $\overline{B V}$ & $B \overline{C V}$ & $\overline{B \nabla}$ & $B \bar{V}$ & 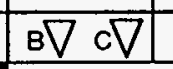 & $\mathrm{BV}$ & $\mathrm{BV}$ & 180 & 290 \\
\hline & $\begin{array}{l}\text { Close Out } \\
\text { Cogement and }\end{array}$ & & & & & & & & & & & & & & \\
\hline$\|$ & Facllly ynd Property & & A) & $\mathrm{Cod}$ & & & & & & & & & & 456 & 250 \\
\hline & Disposstlion & & & & & & & & & & & & & & \\
\hline III & Data Reporting and & & $\bar{A}$ & & & B) & $c \nabla$ & $D \nabla=\sqrt{D}$ & $\mathrm{~F} \nabla$ & & & & & 463 & 860 \\
\hline III) & Archiving & & & & & & & & & & $D D_{E} \nabla_{F} \bigcirc$ & & $c 0$ & & \\
\hline iv & $\begin{array}{l}\text { She Environmential } \\
\text { Comonlance and }\end{array}$ & c) & & वर & ch & c) & $\bar{A} \nabla d \nabla$ & $c \nabla$ & $\mathrm{B} \nabla \mathrm{c}$ & $d V$ & $c \sqrt{ }$ & $\mathrm{cV}$ & $c \nabla$ & 304 & 600 \\
\hline & $\begin{array}{l}\text { Compplannecand } \\
\text { Remediation }\end{array}$ & & & & & All & & & & & 80 & & & & \\
\hline & & & & & & & & & & & & & & & \\
\hline & & & & & & & & & & & & & & & \\
\hline & & & & & & & & & & & & & & & \\
\hline & & & & & & & & & & & & & & & \\
\hline & & & & & & & & & & & & & & & \\
\hline & & & & & & & & & & & & & & & \\
\hline & & & & & & & & & & & & & & & \\
\hline & & & & & & & & & & & & & al Costs: & 1403 & 2000 \\
\hline & $\begin{array}{l}\text { Costs Appear in in : } \\
\text {-EAC-Estimate }\end{array}$ & $\begin{array}{l}\text { Uusands } \\
\text { ompletic }\end{array}$ & $\begin{array}{l}\text { ased on } \\
\text { of Seple }\end{array}$ & $\begin{array}{l}\text { Reporting } \\
r 30,1995\end{array}$ & slem, anc & are cumulat & e for the $F$ & 95 Progral & & & & & & $\nabla_{\text {PLANMED }}$ & \\
\hline & inature of Contractor's $\mathrm{P}$ fof & & & & & & & & 3. Signaturoc & sovernmor & in Rapresentiath & -\& \& Dato & & \begin{tabular}{|l|} 
coMple \\
\end{tabular} & TED \\
\hline & gnature of Contractor's P(p) & Managarerg & $1 \mathrm{ce}$ & ha & & $7 / 1$ & $8 / 90$ & & & & & & & $\bigcup_{\text {RESCHED }}$ & DULED \\
\hline
\end{tabular}




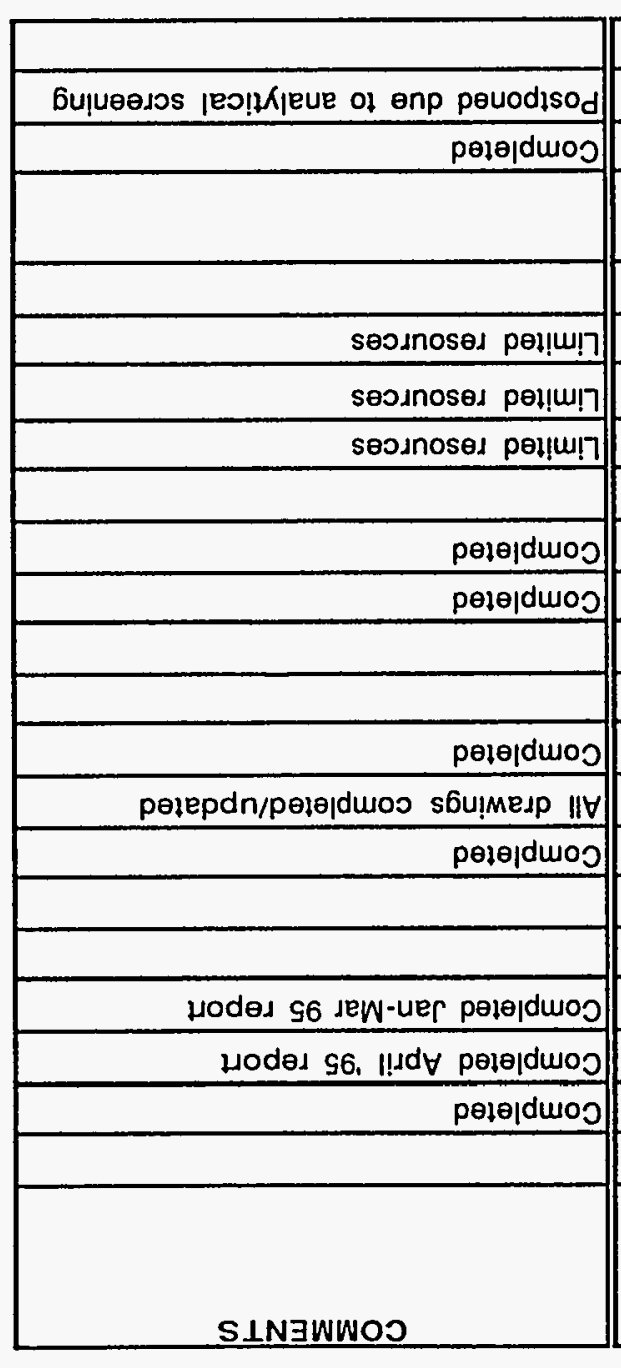

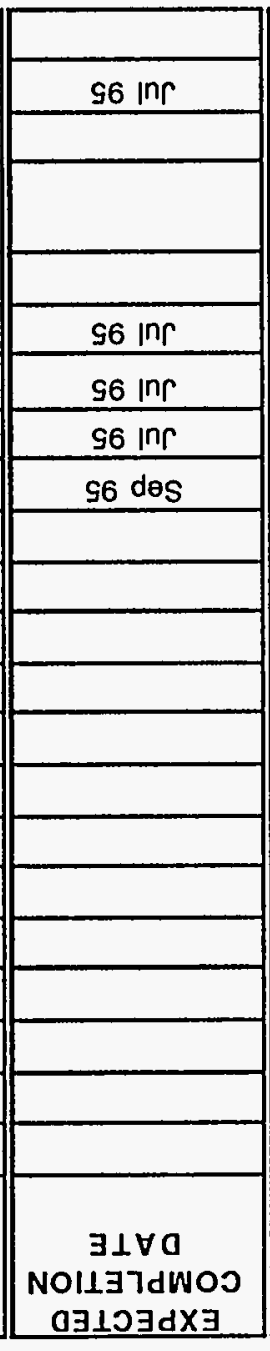

\begin{tabular}{|c|c|}
\hline peleo!̣pu| s甘 & \\
\hline s6 Kew & \\
\hline S6 deW & $969 \theta^{\prime}$ \\
\hline & \\
\hline S6 KeW & \\
\hline$S 6 d d y$ & \\
\hline S6 dd & \\
\hline S6 dew & \\
\hline $969 \theta_{1}$ & $969 \mathrm{~g}^{\mathrm{J}}$ \\
\hline$\forall 6 \wedge \mathrm{ON}$ & D6 $10 \mathrm{~N}$ \\
\hline$t 6500$ & 76000 \\
\hline 16000 & 76000 \\
\hline 16100 & 76100 \\
\hline & \\
\hline pęеग!pu| s & S6 ddV \\
\hline peles!pu| sy & S6 IdV \\
\hline 96 qoy & s6 qa \\
\hline 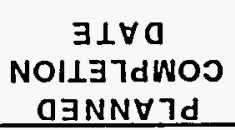 & $\begin{array}{c}\exists \perp \forall a \\
\text { NoI } \perp \exists 7 d w O 0 \\
7 \forall n \perp 0 \forall\end{array}$ \\
\hline
\end{tabular}

\begin{tabular}{|c|c|}
\hline suodey Gu|10!!uow e6jeyos!a deleM RIuluow enss! & $0 \wedge 1$ \\
\hline 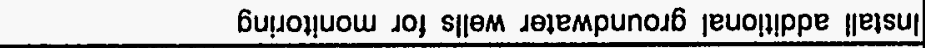 & $\theta \wedge$ \\
\hline 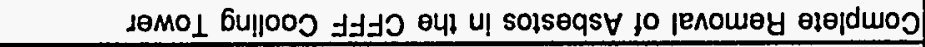 & $\forall \wedge$ \\
\hline 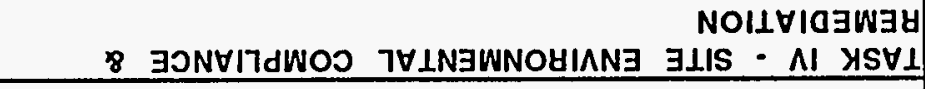 & \\
\hline 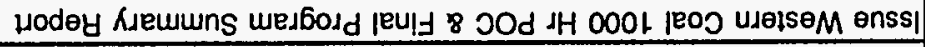 & JIII \\
\hline 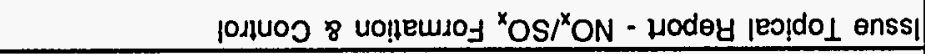 & $\exists$ III \\
\hline 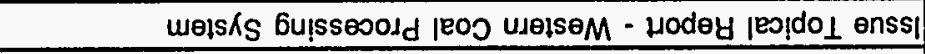 & Q IIII \\
\hline 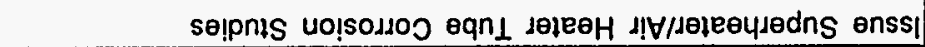 & O॥I \\
\hline 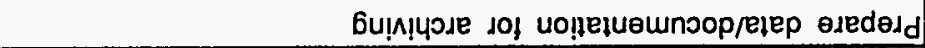 & gIII \\
\hline 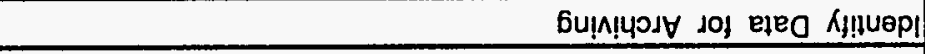 & $\forall$ III \\
\hline 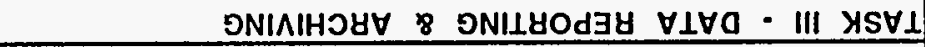 & \\
\hline Kjojuenul Kjeadosd juemusenos jenuuv & OII \\
\hline 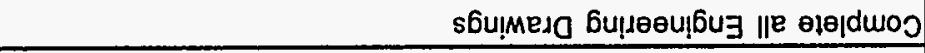 & gll \\
\hline 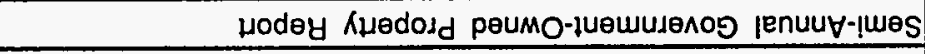 & $\forall \|$ \\
\hline 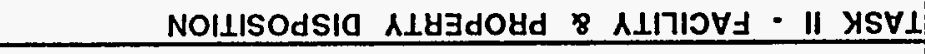 & \\
\hline shodey K Kęueno enss| & 01 \\
\hline syodey R|uluow enss| & 81 \\
\hline 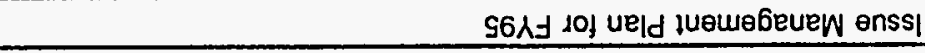 & $\forall 1$ \\
\hline INO $\exists S O 70$ \& IN & \\
\hline Nolldiyos $\exists a$ & ON $\cdot 0 \cdot 1$ \\
\hline
\end{tabular}

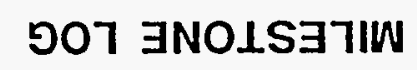


Operation of the MHD Coal-Fired Flow Facility

4. Contractor (Name and Address)

The University of Tennessee Space Institute

Tullahoma, Tennesse日 37388-8897

a

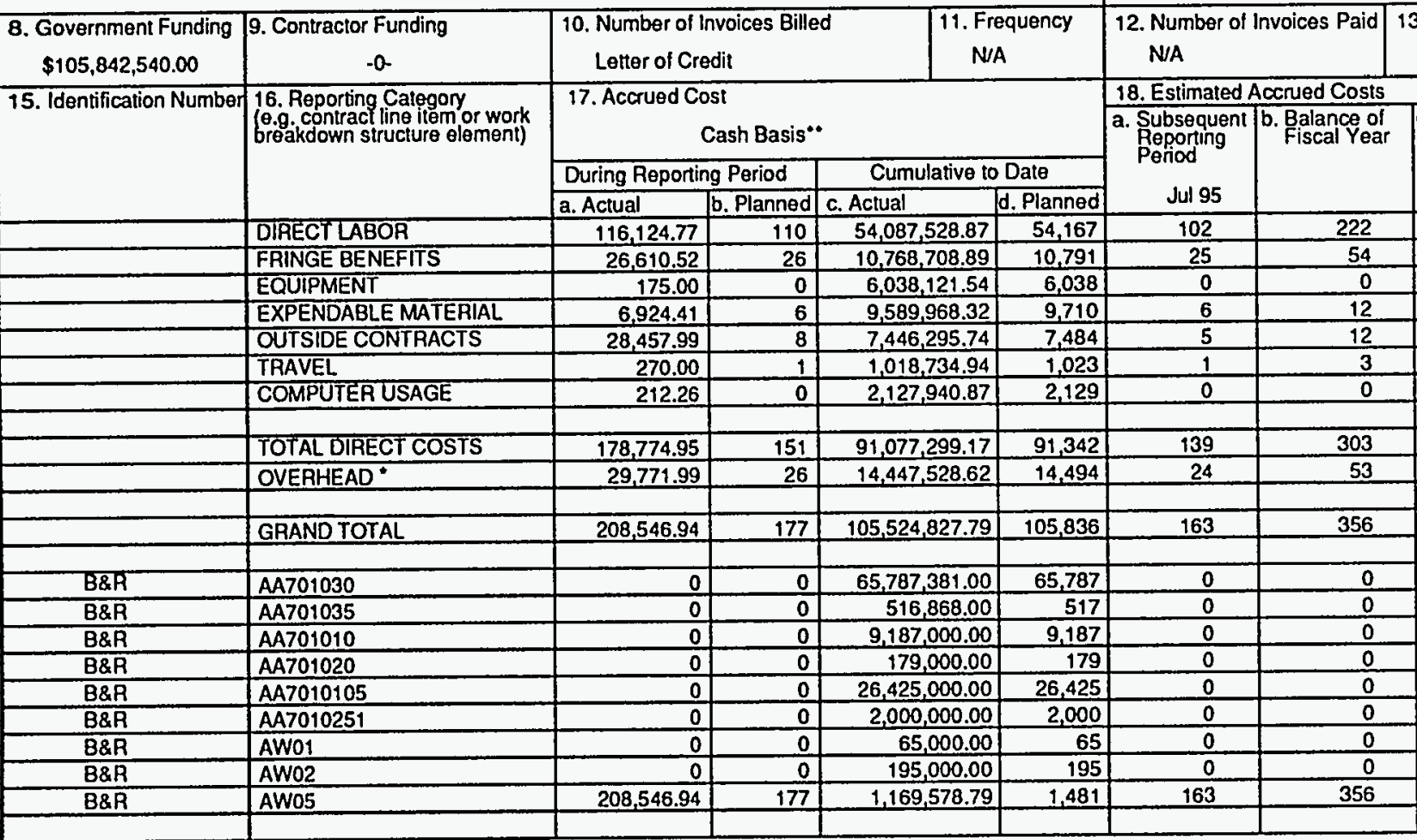

23. REMARKS: : Overhead rate effective $7 / 1 / 94$ is $18 \%$ of TDC less capital equipment, outside contracts over

... Includes orders outstanding costs listed in column 21.

THIS IS A PRELIMINARY REPORT PENDING FINAL CERTIFICATION OF COSTS BY THE CONTROLLER'S OFFICE FOR THE UNIVERSITY'S ACCOUNTING YEAR ENDING JUNE 30, 1995.

25. Signatigre of Contractor's Project Manager and Date

6ignature of Contractor's Authorized Financial Representative \& Date
2. Reporting Period

June 1 through June 30, 1995

5. Cost Plan Date

February 1995

7. Contract Completion Date

September 30, 1995

\begin{tabular}{l|l} 
Tol Total Payment Received
\end{tabular}

$\$ 105,524,827.79$

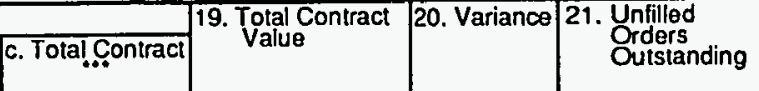

thru 9/30/95

\begin{tabular}{l|l}
\hline 54,515 & 54,170 \\
\hline 10,875 & 10,792 \\
\hline
\end{tabular}

10,875

\begin{tabular}{r|r}
\hline 9,644 & 9,710 \\
\hline
\end{tabular}

\begin{tabular}{l|l}
7,469 & 7,785 \\
\hline
\end{tabular}

1,023

91,692

\begin{tabular}{ll}
91,692 & 91,347 \\
\hline 14,555 & 14,495
\end{tabular}

\begin{tabular}{l|l}
\hline 106,247 & 105,842 \\
\hline
\end{tabular}

\begin{tabular}{|r|r|}
\hline 65,787 & 65,787 \\
\hline 517 & 517 \\
\hline
\end{tabular}

9,187

$\frac{179}{26,425}$

2,4000

195

1,892

\section{lees.}

992

\begin{tabular}{r|r|}
\hline 195 \\
\hline 1,487 \\
\hline
\end{tabular}

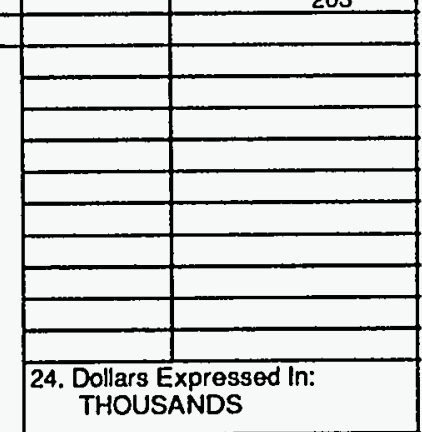

27. Signature of Govemment Tectnical Representative and Date 


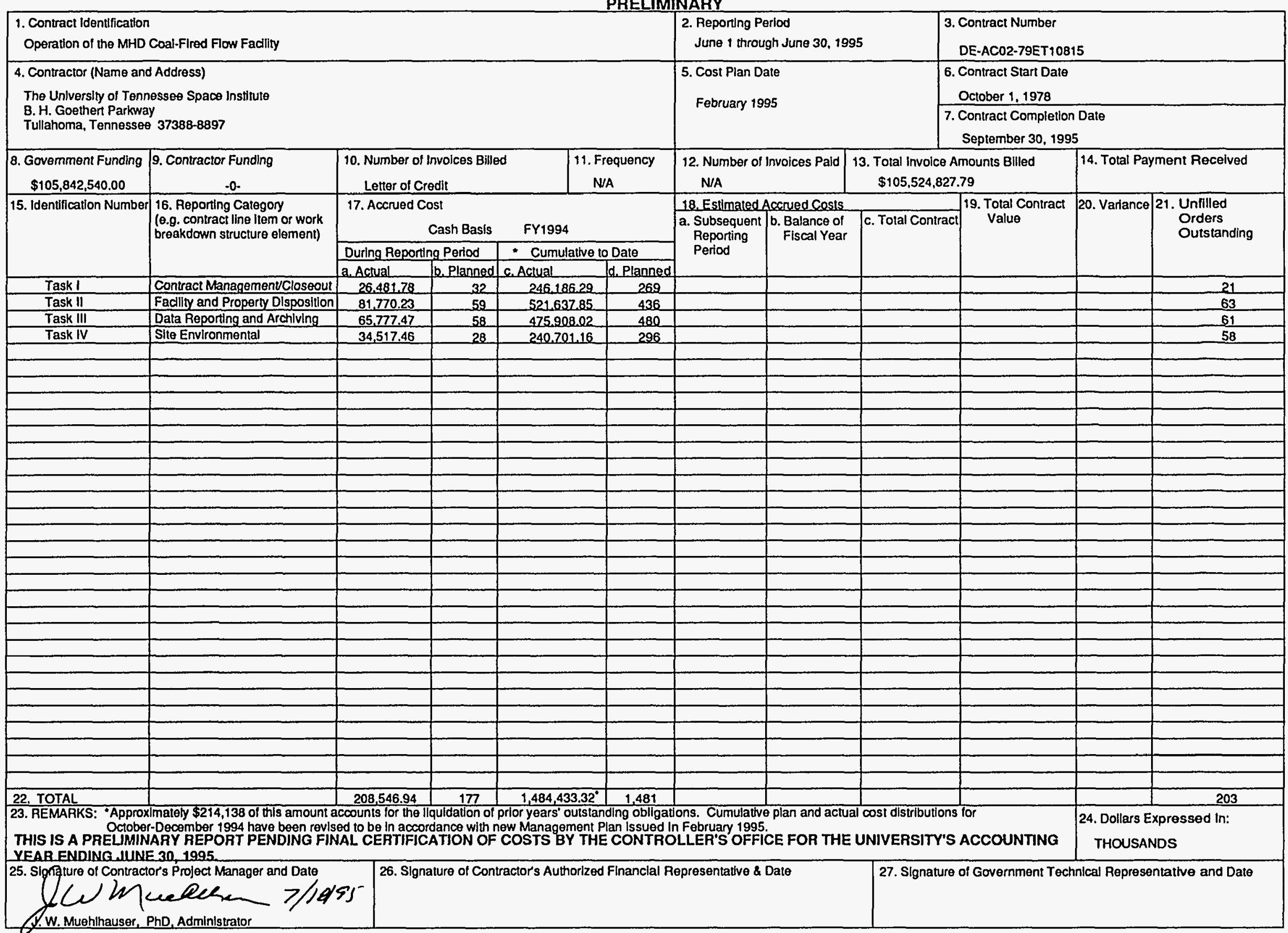

